\title{
Design of Trigger of Foreign Language Audiovisual Products Accounting System
}

\author{
Xuefeng Liu \\ Bohai University, Jinzhou, P.R. China \\ haoxiangchun2005@163.com
}

Keywords: audiovisual products; accounting system; trigger; design

\begin{abstract}
Referential integrity refers to data integrity between two tables, in the process of software development, if cannot ensure referential integrity, will result in disastrous consequences for data. In this paper, based SQL Server database management system designed on the trigger of audiovisual product accounting system, in order to ensure data integrity and provide scientific solutions. Firstly, research the working principle of "INSERT, DELETE, UPDATE" three types of triggers; then, the database structure design, including conceptual structure design and logical structure design; and finally, for audiovisual product accounting system "Account head table" and the "Account detail table "designed three triggers. Contents of this paper, to improve development efficiency of audiovisual product accounting system, to ensure data integrity, and so has an important role.
\end{abstract}

\section{Introduction}

Database integrity includes entity integrity, referential integrity and user-defined integrity. Ensure database integrity is an important task of the database design. Referential integrity refers to a table with a foreign key and another table's primary key data corresponding consistency, to ensure the effectiveness of the contact between the table and table in a database, to prevent loss of data or meaningless data diffusion in the database [1]. Software development process if cannot guarantee referential integrity, will have catastrophic consequences for data. Implement referential integrity usually there are three ways, one is relational constraints of database management system, the second is the application program, and the third is the trigger. Which triggers powerful, able to enforce referential integrity is more complex [2], so that more than one table to add, update, or delete rows, retain a relationship between these tables defined in the software system development is widely used.

The trigger is composed of three parts: events, conditions, actions. When an event occurs and the condition is satisfied, then the action is performed. The use of triggers can allow/restrict changes to the table, automatically derived column, forcing the consistency of the data, providing auditing and log records, prevent invalid transaction processing, start complex business logic, trigger technology has been widely used database system [3]. Audiovisual product refers recorded with the contents of audio and video tapes, records, compact discs and laser discs, including CD, VCD, DVD, DVCD, EVCD, Blu-ray Disc, etc [4]. In recent years, with the rapid development of multimedia technology, more and more audiovisual products in foreign language learning, the use of a computer system to manage audiovisual products is the future trend of development. This article will apply trigger technology in foreign language audiovisual products accounting system, in order to ensure data integrity and provide scientific solutions.

\section{Working Principle of Trigger}

In the execution of DELETE or UPDATE statement, the data rows deleted from the trigger belongs to table, and transferred to the deleted table. The inserted table is used to store a copy of INSERT and UPDATE statements affected rows. In an insert or update transaction, the new rows are simultaneously added to the inserted table and trigger belongs to table. Data operations on the database including the "query, insert, delete, modify," four kinds and others, "query" operation does 
not change the data in the database, there is no impact on the integrity of the database, only "insert, delete, modify," three operations to change the data, so the database management system exist "INSERT, DELETE, UPDATE" three triggers. Specific working principle is shown in Fig. 1 [5].

The working principle of INSERT trigger as shown in Fig. 1 (a), when the trigger belongs to table is inserted a row data, INSERT trigger is fired; The working principle of DELETE trigger as shown in Fig. 1 (b), when the trigger belongs to table is deleted a row data, DELETE trigger is fired; The working principle of UPDATE trigger as shown in Fig. 1 (c), when the trigger belongs to table is modified the data, UPDATE trigger is fired.

\begin{tabular}{|c|c|}
\hline Monitoring INSERT statement & Insert data into the trigger table \\
\hline Execute statements defined by trigger & Generate a temporary table inserted \\
\hline
\end{tabular}

(a) Working principle of INSERT trigger

\begin{tabular}{|c|c|}
\hline Monitoring DELETE statement & Delete data row belongs the trigger table \\
\hline Execute statements defined by trigger & Generate a temporary table deleted \\
\hline
\end{tabular}

(b) Working principle of DELETE trigger

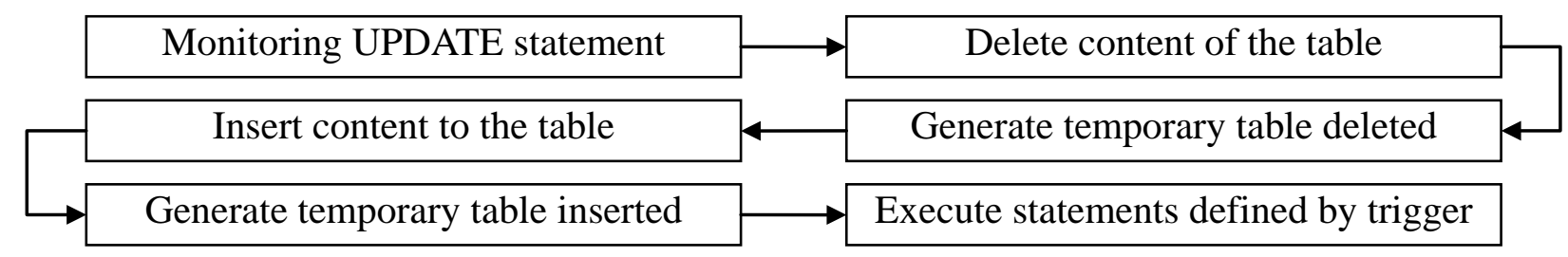

(c) Working principle of UPDATE trigger

Fig. 1. Working principle of trigger

\section{Database Structure Design}

In foreign language audiovisual products accounting system, including the "Account head" and "Account detail" the two entities, "Account head" stores basic information of a kind of audiovisual product, "Account detail" stores detailed information of enter warehouse and out of warehouse, relationship is one to many between the two. A record of "Account head" corresponding many records of "Account detail"; a record of "Account detail" can only correspond to a record of "Account head", the E-R diagram of system as shown in Fig. 2 .

This paper conducts logical structure design based on the SQL Server database management system. In SQL Server, there are two dozen types of data [6], according to the conceptual structure of Fig. 2, grouped into four kinds of data types, namely: character type uses "Varchar", Integer type uses "Int", and decimal type uses "Numeric", date and time type uses "Smalldatetime". With "ProductCode" as an example, the logical structure design of each field is as follows "(ProductCode, Varchar, 10)", where "ProductCode" indicates that the field name, "Varcha" said the field type, "10" indicates the byte number of storage occupied. The conceptual structure design of Fig. 2 convert logic structure design results are as follows:

AccountHeadTable: [(ProductCode, Varchar, 10), (ProductNname, Varchar, 50), (Manufacturers, Varchar, 100), (ProductionDate, Smalldatetime, 4), (MeasurementUnit, Varchar, 20), (CategoryNname, Varchar, 30), (CategoryCode, Varchar, 4), (StorageLocation, Varchar, 100), (BalanceAmount, Int, 2), (BalanceMoney, Numeric, 10,2)].

AccountDetailTable: [(OrderFields, Varchar, 30), (ProductCode, Varchar, 10), (OperateDate, Smalldatetime, 4), (OperateTime, Smalldatetime, 4), (VoucherNo, Varchar, 20), (Summary, Varchar, 100), (Remarks, Varchar, 200), (BusinessPersonnel, Varchar, 30), (OperateName, Varchar, 30), (InAmount, Int, 2), (InPrice, Numeric, 10,2), (InMoney, Numeric, 10,2), (OutAmount, Int, 2), 
(OutPrice, Numeric, 10,2), (OutMoney, Numeric, 10,2), (BalanceAmount, Int, 2), (BalancePrice, Numeric, 10,2), (BalanceMoney, Numeric, 10,2)].

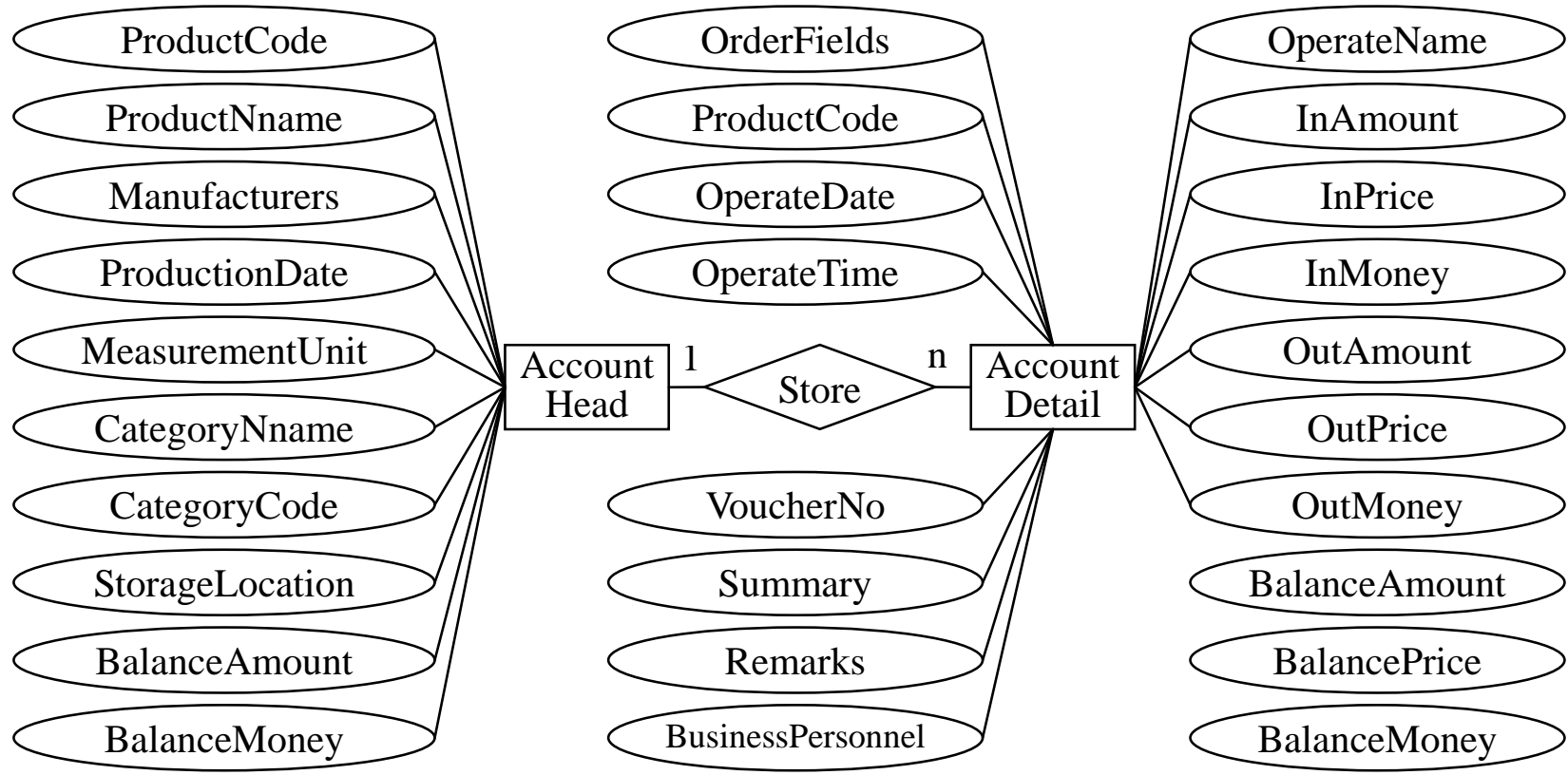

Fig. 2. E-R Diagram of Foreign language audiovisual products accounting system

\section{Trigger Design}

Ensure that foreign language audiovisual products accounting system referential integrity, need to design the following three triggers.

(1) Simultaneously delete data triggers. When the "Account head table" a record is deleted, "Account detail table" at the same time many records are deleted.

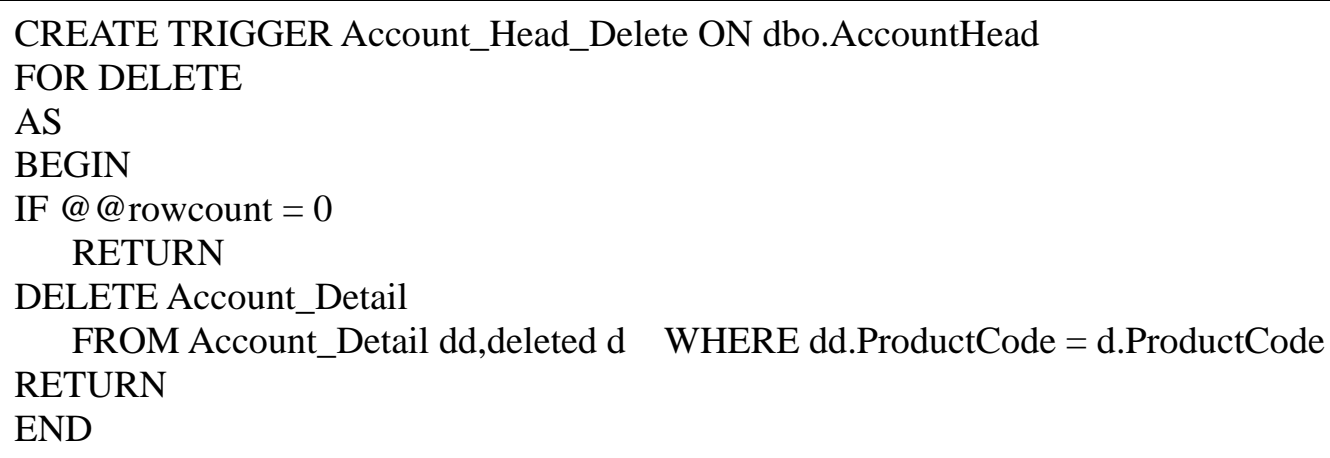

(2) Simultaneously modify the data trigger. When "ProductCode" field content in the "Account head table" is modified, "Account detail table" appropriate "ProductCode" field content is also doing the same modifications.

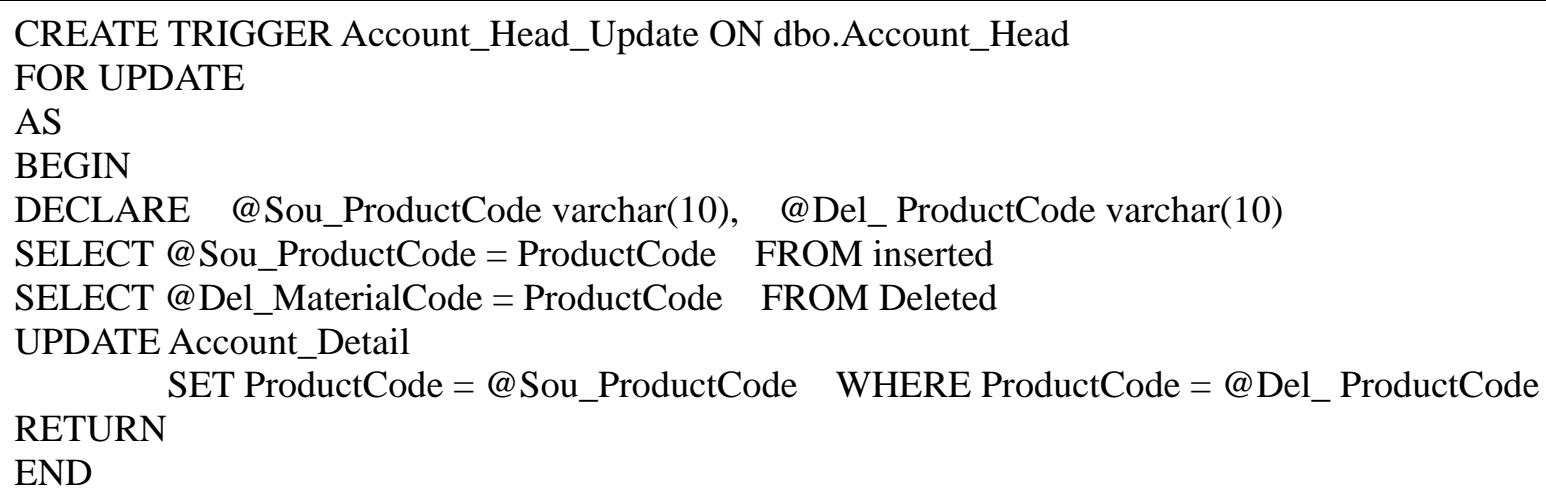


(3) Insert balance data triggers. When the "Account detail table" to insert the data, the balance amount and balance amount will certainly change, will change the data written to the "Account head table".

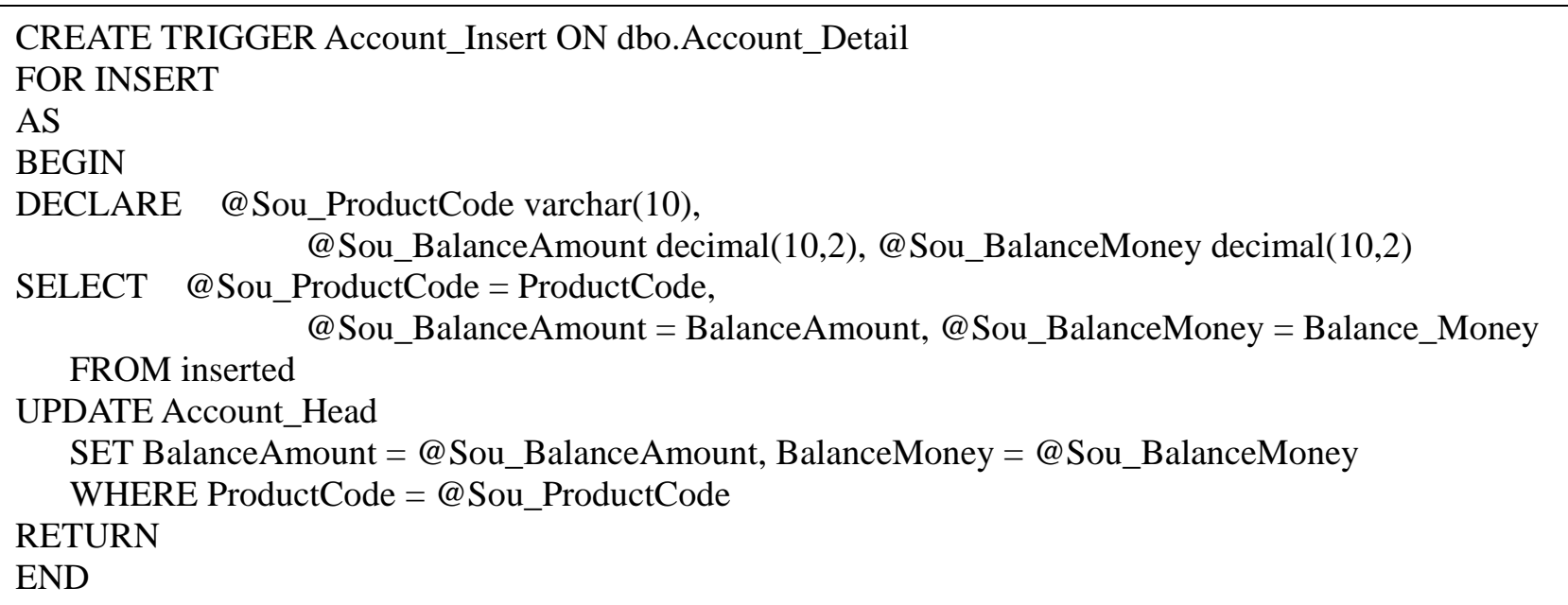

\section{Conclusion}

With all kinds of foreign language learning audio, CD-ROM and other auxiliary electronic books have come out, the use of multimedia learning foreign languages has become a trend, foreign language audiovisual products accounting system to achieve comprehensive management of foreign language audiovisual products, to improve the informatization level of management, improve the utilization efficiency of audiovisual products, improve learners effects play an important role [7]. The research contents of this paper, simplifies database system design and software development. Triggers can maintain row level data integrity, modify data in the database a series of related tables; can compare the data modification state before and after data modification state, and to take measures in accordance with the difference; data modification statement in a table allows multiple triggers, so that the same data modification statements can produce a number of different collaboration [8]. The research contents of this article, to use SQL Server database for software developers, in order to realize referential integrity provides a specific method, and has broad application prospects.

\section{References}

[1] Y. C. Liu, "Implementation Method of SQL Server Reference Integrity for Software Development," Computer Technology and Development, vol. 23, no. 6, pp. 111-114, 2013.

[2] L. J. Zhao, "The Data Referential Integrity of Analysis in SQL Server 2000 Databases," Office Informatization, vol. 16, no. 24, pp. 40-41, 2010.

[3] danielhjd Blog, "The definition and function of the trigger," http://danielhjd.iteye.com/blog/1058341, 2014-12-18.

[4] Baidu Encyclopedia,

"Audiovisual products," http://baike.baidu.com/link?url=UFsUL2aQ-FGYe-xyNuJHam2km8py1nXp9K , 2014-12-18.

[5] F. M. Liang, "SQL Server 2000 database programming," Beijing Hope Electronic Press, 2002.

[6] Robin Dewson, "Beginning SQL Server 2005 for Developers," Apress, 2006.

[7] P. L. Cai, "Publishing significance and research on English vocabulary fast memory books and audio-visual products," Science Technology \& Publication, vol. 24, no. 2, pp. 55-56, 2005.

[8] W. X. Liu, "Further Study on Trigger of SQL Server Database," Computer Technology and Development, vol. 23, no. 10, pp. 48-51, 2013. 